\title{
Exploring the adoption of social media in self-paced physical activity in early childhood education: a case in central China
}

\author{
Jinjin Lu ${ }^{1}$ iD \\ Accepted: 18 October 2021 / Published online: 11 November 2021 \\ (c) Association for Educational Communications and Technology 2021
}

\begin{abstract}
Social media web 2.0 technologies can be adopted as an inclusive method to assist children in enhancing their quality of education for the Sustainable Development Goals (e.g. SDGs 4 and 17). Social media technologies have been documented as low-cost, synchronous, and convenient methods to assist young children, parents, and early childhood (EC) teachers in communication and collaboration. However, few researchers have explored the potential usage of social media tools in enhancing children's physical activities in the early years, particularly during the pandemic period. This article uses the example of WeChat Sports, one of the most famous children's games supported by social media web 2.0, to explore the benefits, challenges, and potentialities of using them in ECE. This research uses semistructured interviews to gain a deeper understanding of the attitudes and perspectives of EC teachers and parents on the potential usage of social media technologies in supporting children's physical activities. The results reveal that most participants had a good understanding of using scientific methods to assist children's physical development, however, they expressed concerns on children's health, privacy, and formal learning in terms of overexposure to social media technologies. Suggestions and implications are also provided for both social media application developers and educators in future research studies.
\end{abstract}

Keywords Social media technologies · Early childhood education · Sustainable development goals · Quality and equality

\section{Introduction}

In the twenty-first century, young children spend significant proportions of their time in learning, entertainment and communication through mobile digital applications ('apps') (Third et al., 2017). To improve the quality of the apps, researchers have been exploring various ways of making apps more informative, affordable, and enjoyable for young children in their daily life (Beckman et al., 2014; Gardner \& Davis, 2014). Previous studies

Jinjin Lu

Jinjin.Lu@xjtlu.edu.cn

1 Academy of Future Education, Department of Educational Studies, Xi'an Jiaotong-Liverpool University, 111 Ren'ai Road, Suzhou Industrial Park, Suzhou 215123, Jiangsu Province, People's Republic of China 
(Gardner \& Davis, 2014; Third et al., 2014) have demonstrated that young people regard social media technologies as affordable, accessible, and efficient tools to obtain immediate information, and the youth believe that they are able to access personalised information that could be tailored to their individual needs.

Moreover, the Sustainable Development Goals (SDGs) 4 and 17 highlight that the development of quality and inequality of education are essential in the strategic plan of 2030. In the report, sustainable development is defined as an approach 'to ensure that it meets the needs of the present without compromising the ability of future generations to meet their needs (United Nations Division for Sustainable Development, 1992)'. Goal 17 of the SDG also aims to create a more inclusive and cooperative global environment for millennials. It emphasises the importance of using technologies to enhance north and south regional and international cooperation in terms of science, technology, and innovation, and to enhance knowledge sharing on mutually agreed terms (United Nations General Assembly, 2015). Compared with the rapid development of social media tools in natural science fields, the potential adoption of social media technologies in the development of children's physical activities can increase their movement, particularly during the COVID-19 pandemic. To fill this gap, this study attempts to use WeChat Sports as a case study to explore the potential roles in developing children's mental and physical health to improve children's physical development in the Chinese ECE context.

\section{Literature review}

\section{Theoretical framework}

This study is underpinned by 'Mode 2' (Gibbons et al., 1994) and the Community of Practice (CoP) (Wenger, 1998). In The New Production of Knowledge, Gibbons et al. (1994) explored changes in the mode of knowledge production in contemporary society. They are concerned with the social sciences and the humanities, as well as with science and technology. In contrast to 'mode 1', 'mode 2' emphasises the application of the knowledge to solve practical problems, and these practices differ from those that govern Mode 1. In addition, Gibbons et al. (1994) claim that both scientific and non-scientific actors can be engaged in interdisciplinary collaborations. Similarly, Hurni and Wiesmann (2014) claim that interdisciplinary collaborations might not only require researchers to cooperate in various disciplines, but also to strengthen ties between researchers and non-researchers in solving practical problems. Researchers believe that interdisciplinary collaborations will become more important in modern societies as 'significant problems of tomorrow will be systemic problems, which cannot be addressed by any one specialty (Domik \& Fischer, 2011, p. 129)'. To solve these problems, interdisciplinary collaborations are needed to ensure experts can work in a broad community and in organisations that encompass multiple ways of collaborating. Schneidewind et al. (2016) proposed the concept of 'Research in Real-World Labs' and explains that 'real-world labs are built on a trans-disciplinary process understanding of the involved scientific and non-scientific actors, methods facilitating collaboration and knowledge integration as well as suitable project designs (p. 10)'. To achieve this aim, research projects must extend beyond boundaries; that is, they must be undertaken in any place other than restricted research labs. In this context, various kinds of actors could be involved in the innovative research process rather than involving only scientific researchers in the knowledge production process. 
The construction of an online community might be an effective way to help different actors be involved in interdisciplinary (e.g. researchers, volunteers, and civics). Lave and Wenger (1991) believe that CoP is a community that provides an environment for members to share views and perceptions of what they do and what these mean to their lives. Later, Wenger (1998) used a social theoretical perspective to consider the development of a CoP as a process that involves identity development, relationship building, and social structure production (Marques et al., 2016). Although the CoP construction process is dynamic and complex, scholars believe that communication is the key to the success of practice-based designs and collaboration for effective teaching and learning (Pella, 2015). Interactions and ongoing communication are highlighted as follows:

Communities of Practice are groups of people who share a concern, a set of problems, or a passion about a topic, and who deepen their knowledge and expertise in this area by interacting on an ongoing basis (Wenger et al., 2012, p. 4).

CoP confirms that the use of social media technologies has the potential to assist in the establishment of active, sustainable, and capacity-building communities (Wenger, 1998). In recent years, scholars have found that traditional face-to-face communities have been transformed to be online because the online environment could provide more opportunities for citizens to participate and be engaged in a more relaxing and flexible way (Karno \& Hatcher, 2020; Lu, 2020). Social media technologies expand opportunities for participants in scientific projects, with increased ease of data sharing and precise measurements for different ages (Liberatore et al., 2018). For example, a Facebook group was built for those who love to share data in the New Zealand Garden Bird Survey (NZ-GBS) because it is convenient to share, connect, and be engaged in a constant virtual way. In addition to data sharing, social media technologies have been used to enhance students' scientific literacy to improve citizen education (Ambrose-Oji et al., 2014). A study by Merkt et al. (2018) revealed that the younger generation could obtain scientific information via videos on the Internet. Moreover, YouTube has recently been documented as one of the most popular social media tools for individuals seeking science-related information (Metag, 2020), however, due to their efficient and synchronous communication, an increasing number of users prefer using instant social media tools, such as Facebook Messenger and WhatsApp.

\section{Impacts of social media technology on young children's development activities}

Social media technologies have been researched as they have a significant influence on young children's cognitive, mental, and physical development. Researchers believe that social media tools could be beneficial for extending the community network in all kinds of activities in classrooms and out of schools. For example, Vollum (2014) demonstrated that social interaction could positively impact education and that social media could improve young children's social interaction in an educational setting. In his review study, he demonstrated how social media tools have been used to enhance health programs outside the K-12 system, which pushed the adoption of social media in K-12 physical education. In Australia, Fan and Yost (2019) found that both parents and EC teachers were interested in adopting social media tools to facilitate better communication, collaboration, and family involvement. In their research, EC teachers believed that using Twitter and Facebook could be more efficient than other tools to socialize and connect with parents. 
In addition, studies have revealed that social media tools can assist young children from diverse backgrounds in the development of language and literacy skills (e.g. Bus et al., 2020; Mol et al., 2014), connected learning (Vartiainen et al., 2019), and children's highorder thinking (Zawilinski, 2009). Apps have been used as beneficial tools for young children who are marginalised in different social contexts. For example, Griffith et al. (2019) conducted a small experiment with 4-year-old children from low-income families and found that children who played educational games on touch-screen devices improved their literacy and mathematics skills more than the control group that only had access to ageappropriate entertainment software. Furthermore, social media technologies have been widely employed in children's daily lives inexpensively. Researchers have claimed in recent years that social media platforms such as Facebook, Twitter, and YouTube have positively promoted physical health for a lifetime (JOPERD, 2017). More recently, physical activities have been promoted not only by the adoption of social media tools via online platforms but also by incorporating them into digital mobile equipment (Bopp \& Stellefson, 2020; Goodyear et al., 2019). Studies found that wearable digital technologies among the youth are beneficial for improving their understanding of digital-physical activity and health technologies and have been influenced by schools, physical education, sports, family, and peers (Goodyear et al., 2019). Meanwhile, Bagot et al. (2018) claimed that the potential use of mobile and wearable technologies could assist researchers in acquiring the biodata of more children in future STEM projects. A few limitations and concerns about the extent to which children could be engaged in using social media technology during physical activities have also been highlighted. For example, Hargittai et al. (2018) claimed that children's age and their comprehension of research goals, levels of complexity, and individual interest would become barriers for children and adults actively participating in scientific projects online. Similarly, Wong et al. (2014) found that it is more difficult to obtain young children's health data by using social media technologies, such as Facebook and Twitter, for photo and video sharing. Moreover, children's privacy, confidentiality, and professionalism when interacting with them online are challenges for researchers as well. Improper usage may lead to cyberbullying and physician misconduct. Exploring the perspective of the target audience would allow for potentially more effective improvements and efforts via social media.

To summarize, previous studies have demonstrated that adopting social media tools may help children's development physically and mentally by expanding scales of data collection, convenience, and low cost. In addition, researchers have indicated that wide and positive use of social media technologies may be used to enhance young children's physical activities. However, most quantitative studies undertaken have not presented children's physical activities and health conditions by using social media technologies during the lockdown. Furthermore, researchers have not addressed questions about how widely social media tools can be used in some social-cultural contexts with severe censorship, such as China, Iran, and North Korea. All these questions require further exploration.

\section{An adoption of WeChat App among Chinese children's physical development}

Due to the severe media censorship in China, popular social media tools, such as Facebook, Twitter, and YouTube, cannot be used on the Chinese mainland (Lu, 2020). Since 2010, WeChat has grown in popularity in the Chinese social media market. According to Statista (2020), the WeChat app (hereinafter 'app') has over 1000 million active 
monthly users, of whom $90 \%$ are Chinese. Compared with other Chinese apps, it integrates audio, video, text chats, document sharing, and other functionalities into one platform, and supports synchronous, asynchronous, and semi-synchronous interactions in multiple languages (Lu, 2020). In this case, people who work and live in the West also have access to the app. The Chinese have used the WeChat app for multiple purposes, such as online learning, communications, e-pay, entertainment, and cloud sharing.

In the National Outline for Medium and Long-term Education Reform and Development (2010-2020), the Chinese Ministry of Education has emphasised the need for enhancing children's technology skills, particularly their ability to use technology (Ministry of Education, 2010). These requirements are in accordance with the SDG goals of enhancing quality and equity in education to narrow the gap between children from families with different socio-economic statuses. Previous studies have indicated that the WeChat app is beneficial for students' informal learning, teachers' professional development, and community resource sharing in China (Guo et al., 2015; Qi \& Wang, 2018; Wang et al., 2016). More recently, Chinese researchers have focused on how WeChat could assist the youth in improving their physical activities and, consequently, their health. For example, WeChat has been adopted as a novel exercise intervention tool to improve metabolic health and poor behaviours among overweight and obese children (Fang et al., 2019). A skipping rope was used as the main interventive physical activity in the program and this explorative exercise protocol demonstrated that exercise had substantial health benefits, including reduced metabolic disease risk and body fat (Fang et al., 2019). During the lockdown, individuals' health information such as daily steps, weight, and regular types of exercise were collected via WeChat in Shanghai, China (Ding et al., 2021). The results indicated that the elderly and more educated individuals were insufficiently active during the lockdown in 2020. In addition, this largescale study provided first-hand data to the government to guide individuals to gradually undertake exercise after the reopening in China. A recent Chinese health survey also reveals people's belief that 'autonomous health education via WeChat is a useful tool to address the current shortcomings in health education' (Zhang et al., 2017, p. 71), although it currently cannot be tailored to individuals.

Amid the pandemic, it is critical to raise citizens' awareness of physical health, particularly for children. The WeChat Sports app was initially developed by Tencent, a local Chinese Internet-based platform company. In the last five years, the WeChat Sports app has attracted many users owing to its syndromic and intergenerational friendly interfaces. The app has been developed and expanded to the community to assist the public in performing exercises, particularly during the pandemic period. The app could be compatible with both Android and IOS systems. In this study, the development of the app interface in the first phase and its essential interactive functions are presented in Fig. 1 and Fig. 2.

The primary focus of the first phase of the research was to develop a thorough understanding of the willingness, perceptions, and concerns of the two key stakeholders in the potential usage of social media technologies in terms of their effectiveness, confidentiality, and proficiency in assisting children in future interdisciplinary projects. To achieve this research aim, we attempted to answer the following two questions:

1. What were the perceptions of EC teachers and parents about their experiences using the WeChat Sports app and potential social media applications?

2. What are the implications of using social media tools for $\mathrm{CoP}$ building for young children in potential interdisciplinary projects? 


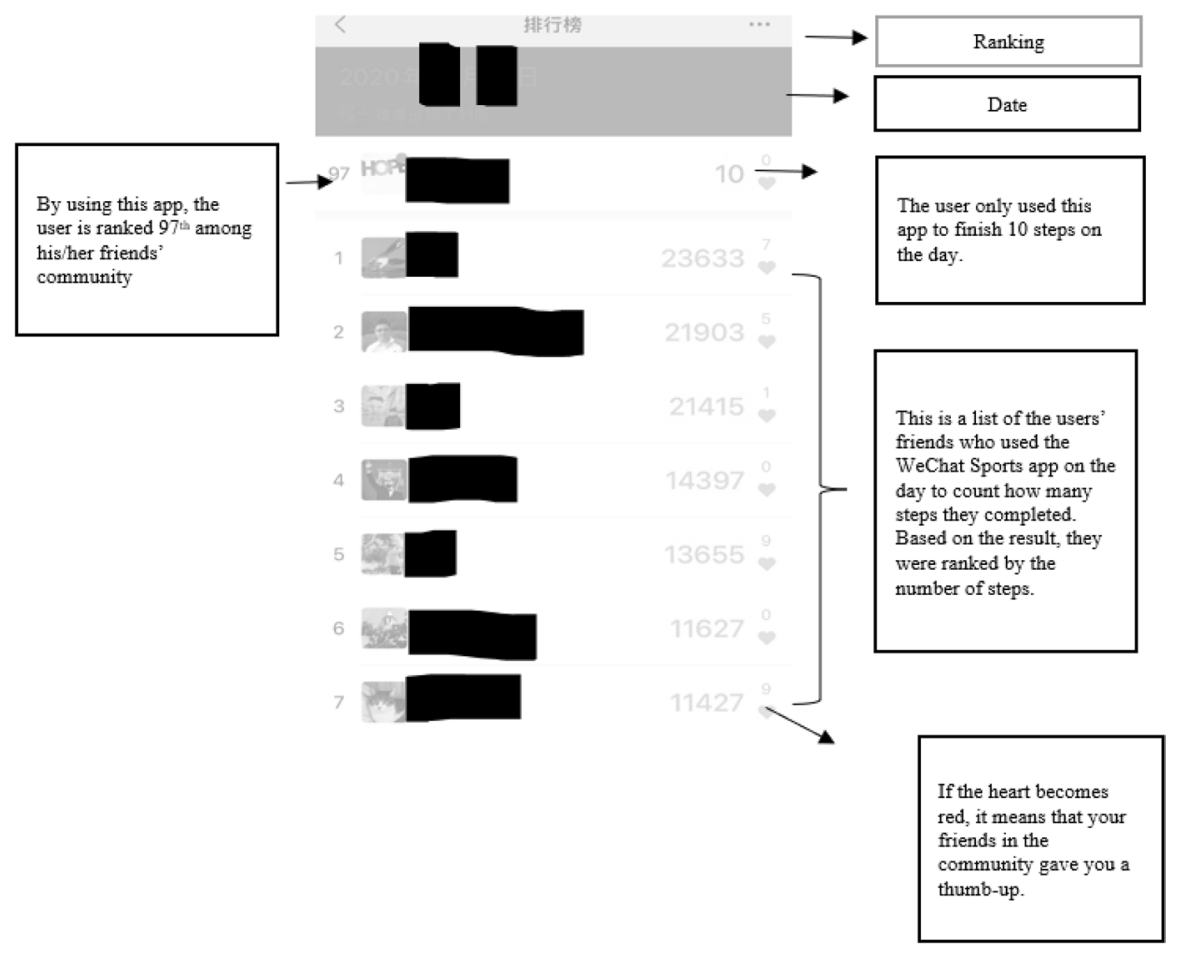

Fig. 1 The interface of the WeChat Sports app

\section{Methods}

\section{Data collection}

After obtaining ethics approval from X University, the research assistant used the WeChat platform to advertise the project flyer, including the research information, online consent forms, and the principal researcher's contact information. After signing the online consent forms, the participants were contacted by the research assistant to schedule their interviews (questions in the appendix protocols). All interviews were undertaken online due to the safety control measures during Covid-19. Semi-structured interviews were conducted and recorded by the principal researcher and the research assistant. Each interview lasted between 40-45 min. The principal researcher then used the constructivist grounded theory approach (Charmaz, 2006) and NVivo software Version 12 to transcribe and analyse the recordings. The researcher used a three-step coding approach within the constructivist grounded theory approach. First, the researcher carefully read each line of the interview transcripts to identify the facts, comparing and regrouping the lower categories with the higher sub-themes constantly. Finally, after these processes, the themes emerged from the sub-themes (Charmaz, 2006). 


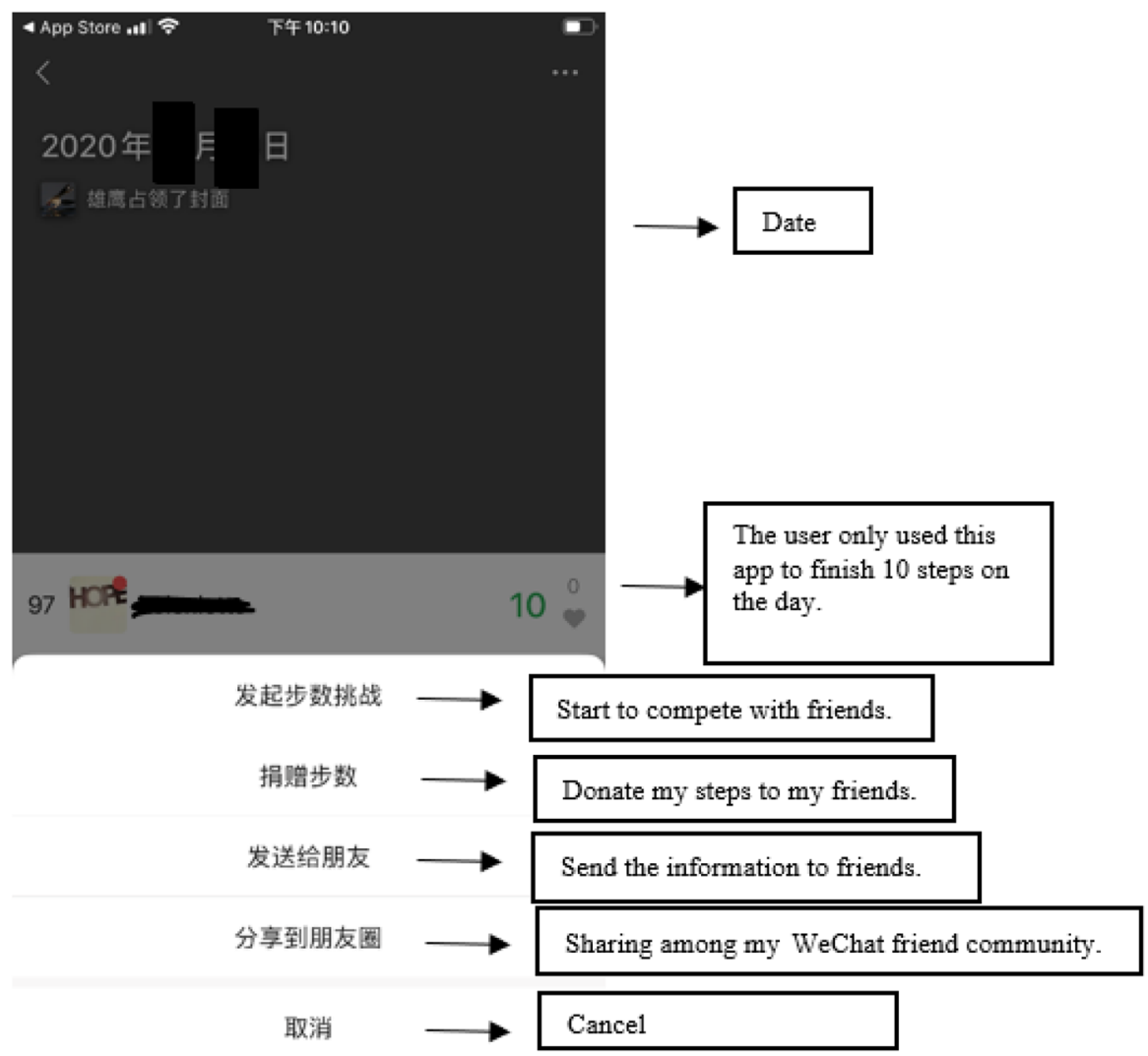

Fig. 2 The functions of the WeChat Sports app

\section{Participants}

This study involved 28 participants from $\mathrm{W}$ city in central China. The participants in the city experienced a long-term lockdown for over eight weeks. There were 10 EC teachers in the urban preschools and 18 parents. All EC teachers were female, indicating that women continue to be the dominant gender in the EC profession. For parents, it seemed that fathers $(n=12)$ evinced more interest than mothers $(n=6)$ to attend the interview. The children of the participating parents ranged in age from 2 to 5 years old. Table 1 presents the demographic data of the two groups of participants.

\section{Results}

The results and findings reported in this paper are textual data for the participants obtained in the first phase of the research. The research results focused on the following two key themes. 
Table 1 Participants' demographic information

\begin{tabular}{|c|c|c|c|c|c|}
\hline \multicolumn{6}{|l|}{ Groups } \\
\hline EC teachers & Age & Gender & $\begin{array}{l}\text { Teaching experi- } \\
\text { ence (Years) }\end{array}$ & Preschool location & Education level \\
\hline P1 & 24 & Female & 1.5 & Urban & $\mathrm{BA}$ \\
\hline $\mathrm{P} 2$ & 25 & Female & 3 & Urban & MA \\
\hline P3 & 27 & Female & 1 & Urban & MA \\
\hline P4 & 23 & Female & 1 & Urban & BA \\
\hline P5 & 29 & Female & 7 & Urban & BA \\
\hline P6 & 30 & Female & 8 & Urban & BA \\
\hline P7 & 48 & Female & 30 & Urban & Diploma \\
\hline P8 & 43 & Female & 23 & Urban & Diploma \\
\hline P9 & 28 & Female & 5 & Urban & BA \\
\hline $\mathrm{P} 10$ & 29 & Female & 7 & Urban & BA \\
\hline Parents & Age & Gender & Jobs & Community location & Education level \\
\hline P11 & 35 & Female & Housewife & Urban & BA \\
\hline $\mathrm{P} 12$ & 37 & Male & IT staff & Urban & BA \\
\hline P13 & 29 & Female & Sales & Suburb & BA \\
\hline P14 & 41 & Male & Civil officer & Urban & MA \\
\hline P15 & 27 & Female & Housewife & Urban & BA \\
\hline P16 & 30 & Female & Self-employed & Urban & BA \\
\hline P17 & 32 & Male & School teacher & Urban & MA \\
\hline P18 & 36 & Male & IT staff & Outskirts & BA \\
\hline 919 & 34 & Male & Self-employed & Urban & BA \\
\hline $\mathrm{P} 20$ & 29 & Male & Taxi driver & Outskirts & Diploma \\
\hline P21 & 26 & Female & Music teacher & Urban & BA \\
\hline $\mathrm{P} 22$ & 34 & Male & Civil officer & Urban & MA \\
\hline P23 & 33 & Male & Cook & Urban & Diploma \\
\hline $\mathrm{P} 24$ & 39 & Male & Self-employed & Outskirts & MA \\
\hline $\mathrm{P} 25$ & 34 & Male & Engineer & Urban & MA \\
\hline P26 & 29 & Female & Nurse & Urban & BA \\
\hline P27 & 30 & Male & Self-employed & Suburb & $\mathrm{BA}$ \\
\hline P28 & 35 & Male & IT staff & Urban & MA \\
\hline
\end{tabular}

- Social collaboration for young children; and

- Parents' concerns

\section{Theme 1: social collaboration for young children}

In this theme, the participants held a positive attitude about the use of social media technology for peer collaboration, information sharing, and family involvement during the lockdown. EC teachers and parents believe that social media technology has great potential to build a CoP for young children in future physical activities. With the assistance of 
social media technologies, EC teachers improved their understanding of young children to strengthen inclusive education in the Chinese ECE context.

\section{Peer collaboration and information sharing}

The participants in this study reported that children would have been provided with morning and afternoon sessions for physical activities at preschools before the lockdown. EC teachers would also invite children's parents to attend family activities for some functions, such as children's birthdays, public holidays, and international children's days. Parents expressed that if they were available, they would like to spend weekends with their children enjoying outdoor activities, such as riding bikes, doing sports, and visiting museums. However, during Covid-19, all of them needed to reconsider their plans. The two groups of participants believed WeChat Sports to be an intergenerationally friendly application that would engage and involve the entire family. During the interaction, children's communication and collaboration skills were enhanced, which was highlighted by parents and teachers. One of the parents, a mother of two children, stated the following:

Lifestyle changes under lockdown. Before the lockdown, the two kids were looked after separately by my parents and my parents-in-law. The children could not see each other every day, so they did not feel very close even when we had meals. During the pandemic period, my husband and I looked after the two boys, aged 3 and 5, and we used this sports app to play with them. The two children's watches also have access to this app so that they do not need to use mobile devices. Boys would like to see whose scores were higher, which represents the more energetic person. The younger one, of course, would lose most of the time, but he started to turn to his brother for help rather than his dad.

(Participant 13, mother of two boys)

She was encouraged to describe the changes in the two boys' ways of communication and collaboration using the app:

Before the lockdown, the two usually punched each other until the little one asked the adults for help. That is the major reason they were separated to be looked after by different grandparents. We found that they did not have a lot in common when they stayed with us on weekends. For example, when the little one asked his brother for help, such as co-drawing and blowing blooms, the bigger one ignored or shouted at him loudly. Therefore, we felt very worried to see the two could not live in harmony. In contrast with the 'unpeaceful time', after the lockdown, the two boys felt very interested in using the app to do exercise with us. The bigger one felt very motivated to help the little one to register online, as he believed that he would obtain higher scores than his brother. After dinner, they were willing to step up and down the porch. All the steps and energy would be recorded synchronically by the apps. (Participant 13, mother of two boys)

One of the EC teachers also claimed that the app increased many children's interest in exercising at home. After the lockdown, she was worried about the children's health and wellbeing. Therefore, she informed the parents that they should consider using the app to play sports at home with the children. After a week, she obtained very positive feedback 
from parents, such as increasing the common interest between family members, being active in finishing tasks, and enhancing communication skills.

\title{
Constructing a potential CoP
}

A large number of responses were obtained regarding the construction of a potential CoP for young children. A father strongly believed that the CoP for young children during the pandemic period was essential for ensuring children's mental and physical health. He commented:

\begin{abstract}
Being an adult, I would be crazy if I were staying at home for a couple of weeks. I have never believed that my kid would be okay if she was caged for a few weeks. But actually, we all experienced it this year. The app, more than a sports game, has offered a choice for kids to be virtually engaged. Children could not see their mates in the preschool, but the app allowed them to be involved in a community to do exercises, share the scores, and give others a thumbs-up. The online encouragement and positive attitudes from peers were much more important than what I told my kid.

(Participant 28, father of one boy).

A mother highly appreciated that the app would allow her son to stay at home during the lockdown. She mentioned that at the beginning of February, she had concerns about the suggestions from EC teachers to ask her to try the app with her kid. She further reported that after a couple of times, she found that her son felt very active in moving physically because he knew that his watch app and my phone could see his energy number and the corresponding scores. She expressed that her son felt very proud of himself if his score was higher than his friends in the community, and he started to show off in front of me.

(Participant 11, mother of one boy).
\end{abstract}

\section{An improvement of understandings of young children}

All the EC teachers reflected that they did not understand what kind of physical games or activities each child enjoys. The app helped them identify the needs of different children in physical games. One of the EC teachers commented that because of the large ratio between EC teachers and children in the city, she felt it was difficult to know exactly what each child enjoys, particularly in physical activities. In addition to their physical health, only the children's behaviour and their activities at preschool, such as dancing, playing Jigsaw, and playing on the swings were evident to her. During the lockdown, she found that, in general, boys displayed more motivation and interest in using the app than girls. The girls sometimes needed to be reminded by their parents to exercise at home. In addition, the number of steps taken by girls was much lower than that of boys. Some EC teachers stated that many girls liked to mimic the dance movements seen on TV shows and favoured a 'roleplay' with parents at home. That is, some girls wore their mother's dresses and imitated the singer's facial expression by swirling and jumping. Meanwhile, parents acted as their fans to cheer them up. One of the EC teachers believed that there was a significant difference between girls and boys in their choices of physical activities. As EC educators, we need to improve our understanding of young children's psychology and physiology.

Both groups of participants held a positive attitude toward the adoption of the app that assisted the children to be involved in a more inclusive environment. Before the lockdown, 
siblings from the same family needed to attend different classes or preschools. In this case, the two children might not have common friends or the same learning environment. Some parents highlighted the importance of providing an inclusive environment for children, as children would be treated equally at preschools. One of the children's fathers said, "My son often talked about Yan's (a boy from the same preschool) dad being a manager and his parents often took him to travel to Europe. I was really concerned that my son would be looked down upon by other preschool mates if it did not improve'. He paused for a while before continuing to comment that after using the app, his son would prefer to talk more about his scores rather than being jealous of other children.

\section{Theme 2: concerns about adopting digital technologies}

The second theme was participants' concerns about children's health and well-being on using social media technologies as potential tools in interdisciplinary projects. Although it received a small number of responses, the references of the three sub-themes highlighted the essential aspects of parents' and EC teachers' concerns.

\section{Children's health and confidentiality}

Both parents and EC teachers had concerns about children's physical and mental health after being exposed to digital devices for a long time. Parents believed that social media tools were beneficial to the family during the lockdown period, however, when children resume preschool, it might be risky if they are addicted to these mobile devices. These perceptions were also echoed by EC teachers, who commented that neither did the parents nor the teachers receive any professional health and medical training to guide them on monitoring the children and guide them on using social media technologies at preschools. One of the parents who had two boys expressed his concerns and said:

My two boys were separated from their mom for two years, as she decided to divorce. She was overseas, so that it is highly likely that my sons would live with me. In the last two years, the little boy was looked after by my parents and the elder one was with me. I was afraid of the older one being addicted to digital games and spending too much time on the screen, so I set the password for all mobile devices, such as the iPad, the iPhone, and desktops. During COVID-19, the app did help my son to stay at home more peacefully and he did not feel so sad without seeing his mom. However, I could not imagine that when everything goes back to normal after COVID-19, what he would do. Without the app, he might yield to play other games, and I was too busy to company him at that moment. I cannot imagine what would happen. In addition, if his little brother knew that he was involved in using these technologies, he might learn from the older one, and that would be a mess.

(Participant 20, father of the two boys)

The personal data that children are able to share with others might be another concern for parents and EC teachers. One of the EC teachers said:

Currently, not all preschools can afford to have free WIFI in every classroom. If children need to be involved in interdisciplinary projects by adopting social media technologies, an internet connection would be an issue. Even for those who could afford 
to have Internet access in the classrooms, the EC teachers could not guarantee that the children could use technologies appropriately to protect confidential information, such as preschool locations, business logos, and teachers' personal information. If business data are not secured, EC teachers may have more consequences.

(Participant 3)

\section{The future development of the App}

EC teachers and parents all held a positive attitude towards the adoption of the app during the lockdown, although many expressed concerns about the function of app developers in terms of its potential use in potential interdisciplinary projects. The participants identified the app's benefits in their lives, and also stated that most children need to wear a watch with synchronous data when they undertook physical exercise. If the battery ran out or the parents forgot to synchronise the data with the phone app, EC teachers and parents would not have any records. This may be detrimental. In addition, the children in the community could also keep a track of their steps and energy scores for that day. In this situation, most parents need to carefully check the battery, mobile devices, and apps before the children start to move around. Most parents suggested that the programme developers might need to consider lighter and more convenient sim cards that could easily be attached to the children's hair bands, clothes buttons, and shoelaces.

\section{Discussion}

This paper highlights the vital role of social media technology in facilitating children's mental and physical development and the importance of effective communication and collaboration between family members, EC teachers, and peers during COVID-19. It needs to be recognised that the app assisted parents and EC teachers in communicating effectively during the lockdown. The two groups of participants perceived the benefits of the WeChat Sports App during COVID-19 to enhance the communication and connections between peers, parents, children, and EC teachers. The research results indicate that young children are able to use different technologies, particularly easily monitored apps. This result supports the Chinese National Outline for Medium and Long-term Education Reform and Development (2010-2020), which states, 'Attention shall be given to the revolutionary impact of information technology on education development' (Ministry of Education, 2010, p. 41). Digital citizens are born into a world with mobile devices, which they cannot avoid using in their daily lives. In this scenario, EC teachers and parents need to consider effective strategies to guide children on how and when to use technology to benefit their lives.

Most participants did not have a strong positive attitude towards utilising the app as a potential tool to construct a probable CoP to support future interdisciplinary projects. They believed that the WeChat Sports app was used as a potential tool to develop children's physical health during pandemic COVID-19. However, following COVID-19, there have been many uncertainties associated with using the app in preschools, such as a shortage of qualified teachers and ethical issues, which would become barriers to using social media tools in the local context. This finding is consistent with previous studies that demonstrated the limitations of interdisciplinary collaboration in ECE (Ding et al., 2021; Fang et al., 2019; Lee \& Lu, 2020). EC teachers and parents were also concerned about 
children's health, data confidentiality, and individual ability. This outcome differs from what researchers expected, which was that participants would find it easy to manage and upload data in interdisciplinary ECE projects (Hansen, 2017). Even though the app could benefit children's physical activities and their potential use to construct a CoP, it is unclear who would manage and provide in-kind guidance for young children during COVID-19 if they have difficulties while using them. In the Chinese context, Chinese parents usually have a high expectation of children's formal learning at schools because academic performance is only evaluated by examination scores (Chen et al., 2020; Lu, 2019). For a long time, EC teachers and parents have paid little attention to children's physical activities and communicative development, particularly, without any support outside of schools. In this case, the adoption of social media technology to help children, families, and EC teachers become involved in a community to enhance interdisciplinary projects still has a long way in the Chinese context.

Both parents and EC teachers believed that the app could help children live in a more inclusive and equitable environment. Individuals may benefit from the app by gaining the same opportunity to play and learn from each other. Even individuals who might be introverted could start communicating with their peers online. This result is also reflected in SDG Goal 4, which aims to ensure inclusive and equitable education and promote lifelong learning for all. It advocates that '.. . to eliminate gender and wealth disparities and achieve universal access to a quality higher education (United Nations Development Programme, 2020, p. 1). However, there is still a long way to go to achieve the goal of quality education.

In China, many preschool teachers do not have many opportunities to communicate and connect with academics, scientific staff, and EC consultants. Consequently, the gap between theory and practice might lead to insufficient pedagogical knowledge. Participants' perceptions indicated that it is essential to enhance the quality of education and teachers' development programs in terms of the integration of formal and informal learning designs in less developed cities and regions (Ministry of Education, 2010). Meanwhile, these issues might push decision-makers to consider speeding up the legislation of the ECE law in China (Li \& Lu, 2018). With the policy and ECE law in place, the hierarchical levels of EC governors might have a more systematic way to deal with EC teachers' training, professional development strategies, evaluations, and funding allocation.

All participants held a positive attitude towards the adoption of social media technologies to help young children enhance their physical activities to construct a CoP in the future. Although the app was developed and was only explored during the lockdown, parents highlighted that the prospective CoP model was constructed based on the following model (Wenger, 1998). Figure 3 further illustrates these details.

The CoP not only allows children at the centre of the learning to communicate and share their information, but it also benefits family members and EC teachers to enhance their understanding of individual characteristics. This cannot be easily found in the traditional face-to-face setting at preschools, which supports previous studies. Although the CoP could be constructed by using the app for young children, the design flaw presented an interesting implication for the app developers, which has not been examined in previous studies in the local context. These endeavours assist developers in thinking about ways to create light and easy app-based products for children to wear rather than watches. In addition, the app needs to be easy for educators, academics, and families to access to develop a collaborative interdisciplinary project. Finally, there is a pedagogical implication for the EC programme in tertiary universities in China. It calls for more interdisciplinary collaboration and knowledge sharing to develop ECE programs rather than focusing on scientific disciplines (Gibbons et al., 1994). 
Fig. 3 A potential CoP constructed by using the WeChat Sports app

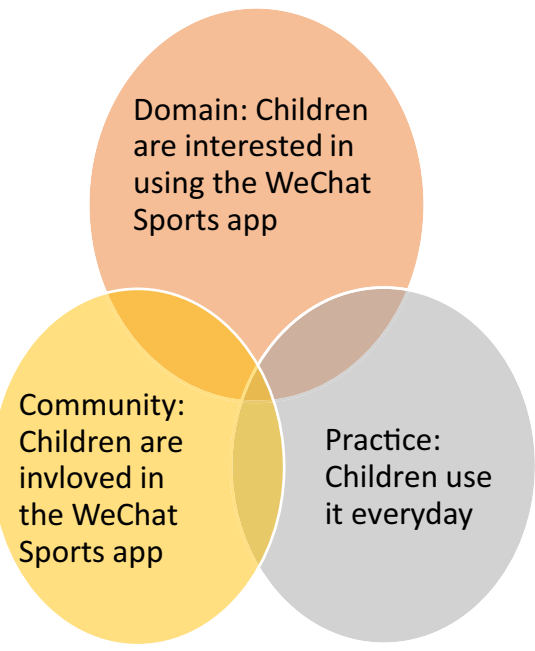

\section{Conclusion}

This paper presents an exploratory study that investigated the potential of using social media technologies to improve children's physical activities, and consequently to construct a potential $\mathrm{CoP}$ in the improvement of interdisciplinary projects in ECE. All participants held positive attitudes towards the adoption of social media technologies upon the development of children's physical activities during the lockdown. The EC teachers and parents in this study recognised the importance of effective communication and collaboration between children, parents, and EC teachers using social media technologies during COVID-19. However, participants also addressed the concerns and issues of insufficient professional development skills and pedagogical knowledge for cohorts of EC teachers, parents, and children, in addition to questioning the ethics of actively involving children in a community after the pandemic. Consequently, the participants did not have a positive attitude towards adopting social media tools in interdisciplinary projects for young children, at least not in this instance. Proposals should be made to include eye-protective screen materials, monitoring apps to manage children in a long-term exploration of specific apps, and helping parents to set up time schedules to participate in programmes involving digital technology for future studies.

The results revealed that participants had a lower level of scientific literacy, and EC teachers had few opportunities to improve their digital literacy for educational purposes, however, there was a desire to adopt and explore these social media technologies in the future.

The research highlighted that children are extremely interested in using social media technologies to complete a range of social activities, such as peer communication, online support, and sharing information. As a digital generation, they are provided with more opportunities to enjoy education equity, but the quality of education still needs to be improved. In the explorative research, a potential CoP was constructed with the help of technology. Finally, although there were concerns about children's health and well-being because of the prolonged exposure to screens, a supportive professional learning programme may alleviate these concerns to some extent. This paper recommends the construction of an interdisciplinary programme for novice EC teachers to assist them to break 
free from the traditional discipline-focused training model. That is, with interdisciplinary professional and educators' support, EC teachers should obtain skills and enhance understanding of knowledge transmission and knowledge construction.

\section{Appendix}

\section{Interview questions for EC teachers}

(Note: Provide explanations of the social media tools used in your teaching).

1. What is your teaching experience? (e.g. the length of your teaching experience or any internships)

2. In what ways do you think that the WeChat Sports App could support the communication and collaboration between parents, children, and teachers in the lockdown?

3. What are the facilitating factors that may motivate you to introduce the WeChat Sports App during the pandemic?

4. How much do you know about interdisciplinary projects? Please give examples.

5. What are the benefits and barriers that may encourage or discourage the adoption of social media tools in your teaching after the pandemic?

6. To what extent could the WeChat Sports App help the children in your class perform physical activities at home?

7. What are some of the concerns you may have in adopting this digital technology?

8. What purpose would you have for digital technology? (e.g. teaching requirements, communication, community building, etc.).

9. On a scale of one to ten, rate your willingness to adopt social media tools to construct potential interdisciplinary projects in ECE.

\section{Interview questions for parents}

(Note: Provide explanations for the social media tools used by your children in daily life).

1. How many children do you have and who looks after them?

2. In what ways do you think WeChat Sports App could support the communication and collaboration between parents, children, and teachers in the lockdown?

3. What are the major reasons motivating you and your children to use the WeChat Sports App during the pandemic?

4. How much do you know about interdisciplinary projects? Please give examples.

5. What are the benefits/barriers that may encourage/discourage you to facilitate your children in adopting social media tools in their daily life after the pandemic?

6. To what extent could WeChat Sports App help your children perform physical activities at home?

7. What are some of the concerns you may have in adopting this digital technology?

8. What purpose would you facilitate your children to use this digital technology? (e.g. assignments for fun, communication, community building, etc.).

9. On a scale of one to ten, rate your willingness to adopt social media tools to construct potential interdisciplinary projects in ECE? 


\section{Declarations}

Conflict of interest No potential conflicts of interest.

\section{References}

Ambrose-Oji, B., van der Jagt, A. P. N., \& O’Neil, S. (2014). Citizen Science: Social Media as a supporting tool. Retrieved from Edinburgh, UK.

Bagot, K. S., Matthews, S. A., Mason, M., Squeglia, L. M., Fowler, J., Gray, K. et al. (2018). Current, future and potential use of mobile and wearable technologies and social media data in the ABCD study to increase understanding of contributors to child health. Developmental Cognitive Neuroscience, August, 32, 121-129. doi:https://doi.org/10.1016/j.den.2018.03.008. Epub, 121-129

Beckman, K., Bennett, S., \& Lockyer, L. (2014). Understanding students' use and value of technology for learning. Learning, Media and Technology, 39(3), 346-367.

Bopp, T., \& Stellefson, M. (2020). Practical and ethical considerations for schools using social media to promote physical literacy in youth. International Journal of Environmental Research and Public Health, 17(4), 1225. https://doi.org/10.3390/ijerph17041225

Bus, A. G., Neuman, S. B., \& Roskos, K. (2020). Screens, apps, and digital books for young children: The promise of multimedia. AERA Open, 6(1), 1-6.

Charmaz, K. (2006). Constructing grounded theory: A practical guide through qualitative analysis. SAGE

Chen, Y., Huang, R., Lu, Y., \& Zhang, K. (2020). Education fever in China: Children's academic performance and parents' life satisfaction. Journal of Happiness Studies. https://doi.org/10.1007/ s10902-020-00258-0

Ding, D., Cheng, M., Del Pozo Cruz, B., Lin, T., Sun, S., Zhang, L., et al. (2021). How COVID-19 lockdown and reopening affected daily steps: Evidence based on 164,630 person-days of prospectively collected data from Shanghai, China. International Journal of Behavioral Nutrition and Physical Activity, 18(1), 40. https://doi.org/10.1186/s12966-021-01106-X

Domik, G., \& Fischer, G. (2011). Transdisciplinary collaboration and lifelong learning: Fostering and supporting new learning opportunities. In C. S. Calude, G. Rozenberg, \& A. Salomaa (Eds.), Rainbow of computer science (pp. 129-143). Springer.

Fan, S., \& Yost, H. (2019). Keeping connected: Exploring the potential of social media as a new avenue for communication and collaboration in early childhood education. International Journal of Early Years Education, 27(2), 132-142.

Fang, Y., Ma, Y., Mo, D., Zhang, S., Xiang, M., \& Zhang, Z. (2019). Methodology of an exercise intervention program using social incentives and gamification for obese children. BMC Public Health, 19(1), 686.

Gardner, H., \& Davis, K. (2014). The app generation. Yale University Press.

Gibbons, M., Limoges, C., Nowotny, H., Schwartzman, S., Scott, P., \& Trow, M. (1994). The new production of knowledge: The dynamics of science and research in contemporary societies. SAGE.

Goodyear, V. A., Armour, K. M., \& Wood, H. (2019). Young people learning about health: The role of apps and wearable devices. Learning, Media and Technology, 44(2), 193-210. https://doi.org/10.1080/ 17439884.2019.1539011

Griffith, S. F., Hanson, K. G., Rolon-Arroyo, B., \& Arnold, D. H. (2019). Promoting early achievement in low-income preschoolers in the United States with educational apps. Journal of Children and Media, 13(3), 328-344.

Guo, M., Wang, M., \& Sun, H. (2015). A pilot study of using mobile platforms (WeChat and WeLearn) in College English curriculum. Paper presented at the The 11th international conference on intelligent environments, Prague, Czech Republic.

Hansen, A. S. (2017). Co-design with children. How to best communicate with and encourage children during a design process. https://www.ntnu.edu/documents/139799/1279149990/13+Article+Final_ anjash_forsøk_2017-12-07-20-11-11_Co-Design+with+Children+-+Final.pdf/b8dd19c4-d2b1-4322a042-718e06663e13

Hargittai, E., Füchslin, T., \& Schäfer, M. S. (2018). How do young adults engage with science and research on social media? Some preliminary findings and an agenda for future research. Social Media + Society, 4(3), 1-10. https://doi.org/10.1177/2056305118797720 
Hurni, H., \& Wiesmann, U. (2014). Transdisciplinarity in practice. Experience from a concept-based research programme addressing global change and sustainable development. GAIA-Ecological Perspectives for Science and Society, 23(3), 275-277.

JOPERD. (2017). Is social media (Twitter, Facebook, etc.) a positive thing for physical education/health teachers to engage in? Why or why not? Journal of Physical Education Recreation and Dance, 88(6), 63-67. https://doi.org/10.1080/07303084.2017.1331669

Karno, D., \& Hatcher, B. (2020). Building computer supported collaborative learning environments in early childhood classrooms. Educational Technology Research and Development. Education Tech Research Dev, 68(1), 249-267. https://doi.org/10.1007/s11423-019-09686-z

Lave, J., \& Wenger, E. (1991). Situated learning: Legitimate peripheral participation. Cambridge University Press.

Lee, L., \& Lu, J. (2020). Using a Citizen Science approach in early childhood education: A call for strengthening evidence. Cogent Education, 7(1), 1-11. https://doi.org/10.1080/2331186X.2020. 1823141

Li, H., \& Lu, J. (2018). Legislation for early childhood education: A case study of China. Children and Youth Services Review, 86, 32-41.

Liberatore, A., Bowkett, E., MacLeod, C. J., Spurr, E., \& Longnecker, N. (2018). Social media as a platform for a citizen Science Community of Practice. Citizen Science: Theory and Practice, 3(1), 3. https://doi.org/10.5334/cstp.108

Lu, J. (2019). Confucius stands on the London Eye- an auto-ethnographic study. Ethnography and Education, 14(1), 51-64.

Lu, J. (2020). The WeChat public platform: Strengthening HSS academics' global competitiveness in non-English speaking countries (La plataforma pública WeChat: Un refuerzo de la competitividad global de los académicos de humanidades y de ciencias sociales en países de habla no inglesa). Culture and Education, 32(3), 609-620. https://doi.org/10.1080/11356405.2020.1785141

Marques, M. M., Loureiro, M. J. L., \& Marques, L. (2016). The dynamics of an online community of practice involving teachers and researchers. Professional Development in Education, 42(2), 235-257.

Merkt, M., Ballmann, A., Felfeli, J., \& Schwan, S. (2018). Pauses in educational videos: Testing the transience explanation against the structuring explanation. Computers in Human Behavior, 89, 399-410. https://doi.org/10.1016/j.chb.2018.01.013

Metag, J. (2020). What drives science media use? Predictors of media use for information about science and research in digital information environments. Public Understanding of Science, 29(6), 561-578.

Ministry of Education. (2010). The National medium- and long-term educational Reform and development guideline (2010-2020). Beijing. Retrieved from http://www.moe.gov.cn/moe_2792/moe_ 2987/s3501/index.html

Mol, S. E., Neuman, S. B., \& Strouse, G. A. (2014). From ABCs to DVDs: Profiles of infants' home media environments in the first two years of life. Early Child Development and Care, 184(8), 1250-1266.

Pella, S. (2015). Pedagogical reasoning and action: Affordances of practice-based teacher professional development. Teacher Education Quarterly, 42(3), 81-101.

Qi, G. Y., \& Wang, Y. (2018). Investigating the building of a WeChat-based community of practice for language teachers' professional development. Innovation in Language Learning and Teaching, 12(1), 72-88.

Schneidewind, U., Singer-Brodowski, M., Augenstein, K., \& Stelzer, F. (2016). Pledge for a transformative science: A conceptual framework. Wuppertal Papers 191. (Discussion paper)

Statista. (2020). Number of monthly active WeChat users from 2nd quarter 2011 to 2nd quarter 2020. Retrieved from https://www.statista.com/statistics/255778/number-of-active-wechat-messengeraccounts/

Third, A., Bellerose, D., Dawkins, U., Keltie, E., \& Pihl, K. (2014). Children's rights in the digital age: A download from children around the world. Young and Well Cooperative Research Centre: Abbotsford, Vic. Australia

Third, A., Bellerose, D., De Oliveira, J. D., Lala, G., \& Theakstone, G. (2017). Young and online: Children's perspectives on life in the digital age. Sydney University.

United Nations Division for Sustainable Development (1992). Agenda 21. Brazil: United Nations.

United Nations Development Programme. (2020). Goal 4: Quality education. Retrieved from https://www. undp.org/content/undp/en/home/sustainable-development-goals/goal-4-quality-education.html

United Nations General Assembly. (2015). Transforming our world: The 2030 agenda for sustainable development

Vartiainen, H., Leinonen, T., \& Nissinen, S. (2019). Connected learning with media tools in kindergarten: An illustrative case. Educational Media International, 56(3), 233-249. https://doi.org/10.1080/09523 987.2019.1669877 
Vollum, M. J. (2014). The potential for social media use in K-12 physical and health education. Computers in Human Behavior, 35, 560-564.

Wang, Y., Fang, W.-C., Han, J., \& Chen, N.-S. (2016). Exploring the affordances of WeChat for facilitating teaching, social and cognitive presence in semi-synchronous language exchange. Australasian Journal of Educational Technology, 32(4), 18-37.

Wenger, E. (1998). Communities of practice: Learning, meaning, and identity. Cambridge University Press.

Wenger, E., McDermott, R., \& Snyder, W. (2012). A guide to managing knowledge: Cultivating communities of practice. Harvard Business School Press.

Wong, C. A., Merchant, R. M., \& Moreno, M. A. (2014). Using social media to engage adolescents and young adults with their health. Healthcare, 2(4), 220-224.

Zawilinski, L. (2009). HOT blogging: A framework for blogging to promote higher order thinking. Reading Teacher, 62(8), 650-661.

Publisher's Note Springer Nature remains neutral with regard to jurisdictional claims in published maps and institutional affiliations.

Dr. Jinjin Lu has over 15 years of international experience in educational research and professional roles in education. Helen obtained her M.A. from the University of Wisconsin in the U.S.A and her PhD in the Faculty of Education at the University of Tasmania. After she finished her PhD, Helen successfully completed a range of education research projects in interdisciplinary teams in Tasmania and New South Wales in Australia. She has developed her professional and academic skills in China, Europe, USA and Australia and her leadership skills as a leading researcher. 